\title{
NONCONNECTED MODULI SPACES OF POSITIVE SECTIONAL CURVATURE METRICS
}

\author{
MATTHIAS KRECK AND STEPHAN STOLZ \\ We would like to dedicate this paper to F. Hirzebruch on the occasion of his 65th birthday.
}

Wir widmen diese Arbeit unserem verehrten Lehrer F. Hirzebruch aus Anlaß seines 65. Geburtstages. Wir hatten beide das Glück, bei ihm studieren zu dürfen, der eine als Doktorand und der andere als Diplomand. Es ist schwer, in Worte zu fassen, wieviel wir Herrn Hirzebruch verdanken. Aber eines möchten wir besonders hervorheben. Herr Hirzebruch hat durch seine ganze Persönlichkeit in uns die Liebe zur Mathematik geweckt. Dafür wollen wir ihm von Herzen danken.

\section{INTRODUCTION}

Very little is known about the question of existence and classification of Riemannian metrics of positive sectional curvature on a closed manifold $M$. The only known simply connected manifolds of dimension $>24$ admitting such a metric are the spheres and projective spaces over $\mathbb{C}$ or $\mathbb{H}$. On the other hand the only known obstructions are the vanishing of the $\hat{A}$-genus [Li], or more generally the vanishing of the $\alpha$-invariant [Hit], and the Betti number bound [Gr].

As far as classification is concerned, the first question is about the number of connected components of the moduli space of such metrics. We denote the space of Riemannian metrics on $M$ with positive sectional curvature by $\mathfrak{R}_{\text {sec }}^{+}(M)$. The group of diffeomorphisms $\operatorname{Diff}(M)$ acts on this space by pulling back metrics. The orbit space $\mathfrak{R}_{\text {sec }}^{+}(M) / \operatorname{Diff}(M)$ is the moduli space of Riemannian metrics with positive sectional curvature. In dimensions 2 and 3 this space is connected. In dimension 2 this follows from uniformization theory. Also in dimension 3 this is related to a sort of geometrization theory. Hamilton [Ha1] has studied the so-called Ricci flow, which has the remarkable property of deforming a positive Ricci curvature metric into a metric of constant positive sectional curvature. If the original metric has positive sectional curvature metric, it stays in this category during the deformation [Ha2, compare §5.2]. Combined with the classification of 3-dimensional Euclidean space forms [CS,

Received by the editors July 20, 1992.

1991 Mathematics Subject Classification. Primary 53C20.

Key words and phrases. Positive sectional curvature, positive Ricci curvature, index of the Dirac operator.

The second author was partially supported by NSF Grant DMS-9002594 and the Max-PlanckInstitut in Bonn. 
p. 778] this implies that the space is connected.

In this paper we will prove that corresponding results about $\mathfrak{R}_{\mathrm{sec}}^{+}(M) / \operatorname{Diff}(M)$ do not hold in higher dimensions in general.

Theorem 1.1. There are closed manifolds for which $\mathfrak{R}_{\mathrm{sec}}^{+}(M) / \operatorname{Diff}(M)$ is not connected.

Similarly, we will investigate the moduli space $\mathfrak{R}_{\mathrm{Ric}}^{+}(M) / \operatorname{Diff}(M)$ of Riemannian metrics with positive Ricci curvature. Again in dimension $\leq 3$ this space is connected by the same argument as above. We will prove that in higher dimensions this moduli space can even have infinitely many components.

Theorem 1.2. There are closed manifolds for which $\mathfrak{R}_{\mathrm{Ric}}^{+}(M) / \operatorname{Diff}(M)$ has infinitely many connected components.

Comparing these results with the situation in dimensions 2 and 3 there are two possible reasons why the moduli space is not connected in higher dimension. Either one cannot deform the metric into a "standard" form or there is a discrepancy between the isometry classification of these standard metrics and the diffeomorphism classification of the underlying manifolds.

To obtain the manifolds of Theorems 1.1 and 1.2 we study two well-known families of Riemannian manifolds of dimension 7 with positive sectional resp. Ricci curvature. The first are the Wallach spaces, homogeneous spaces of the form $G / H$, where $G \cong S U(3)$ and $H \cong U(1)$, which carry homogeneous positive sectional curvature metrics by a result of Aloff and Wallach [AW]. The second family consists of homogeneous spaces of the form $G / H$, where $G \cong S U(3) \times S U(2) \times U(1)$ and $H \cong S U(2) \times U(1) \times U(1)$, which were studied by Witten [Wi]. Both classes of homogeneous spaces are total spaces of $S^{1}$ bundles, in the first case over the flag manifold $S U(3) / T, T$ the maximal torus, and in the second case over $\mathbb{C P}^{2} \times \mathbb{C P}^{1}$ where $\mathbb{C P}^{n}$ is the complex projective $n$-space. Wang and Ziller showed that the latter total spaces admit metrics of positive Ricci curvature, in fact Einstein metrics with positive Einstein constant [WZ].

In both theorems we actually prove a stronger result, namely we find positive sectional (resp. Ricci) metrics which are in different components of $\mathfrak{R}_{\text {scal }}^{+}(M) /$ $\operatorname{Diff}(M)$, the moduli space of positive scalar curvature metrics. We distinguish these components by a $\mathbb{Q}$-valued invariant $s$ which is defined for positive scalar curvature metrics on closed $(4 k-1)$-dimensional spin manifolds $M$ with vanishing real Pontrjagin classes. As a corollary of the construction of $s$ we see that for every such $M(k>1)$ with vanishing $H^{1}(M ; \mathbb{Z} / 2)$ the moduli space $\mathfrak{R}_{\text {scal }}^{+}(M) / \operatorname{Diff}(M)$ has infinitely many components (Corollary 2.15 ), provided that $\mathfrak{R}_{\text {scal }}^{+}(M)$ is not empty. We note that by a result of the second author the space $\mathfrak{R}_{\text {scal }}^{+}(M)$ for a simply connected closed spin manifold $M$ of dimension $\geq 5$ is not empty if and only if the Atiyah invariant $\alpha(M)$ vanishes [St]. This invariant vanishes for $(4 k-1)$-dimensional manifolds and hence for every closed simply connected spin manifold of dimension $4 k-1, k>1$, with vanishing real Pontrjagin classes the moduli space $\mathfrak{R}_{\text {scal }}^{+}(M) / \operatorname{Diff}(M)$ has infinitely many components. Previous work of Hitchin [Hi, Theorem 4.7] and Carr [Ca, 
Theorem 4] shows that $\mathfrak{R}_{\text {scal }}^{+}(M)$ is not connected in many cases, but the result about the moduli space is new as far as we know. We want to stress that in our opinion Theorems 1.1 and 1.2 are deeper results than the last-mentioned result since it is much harder to construct positive sectional (resp. Ricci) curvature metrics.

The organization of the paper is as follows. In $\S 2$ we define the invariant $s$ for positive scalar curvature metrics on certain $(4 k-1)$-dimensional manifolds. This invariant is an absolute version of a relative invariant introduced by Gromov and Lawson. In $\S 3$ we describe the two families of positive sectional (resp. Ricci) curvature manifolds mentioned above and use the invariant of $\S 2$ to prove Theorems 1.1 and 1.2. The main technical tool is the calculation of our invariant for $S^{1}$-equivariant metrics with totally geodesic fibres on total spaces of principal $S^{1}$-bundles (Theorem 3.11). This result is proved in $\S \S 4$ and 5 , with $\S 5$ providing the calculation of the $\hat{A}$-genus of fibre bundles with fibre $\mathrm{CP}^{2}$ and structure group $U(3)$.

\section{AN INVARIANT FOR POSITIVE SCALAR CURVATURE METRICS}

In this section we define a $\mathbb{Q}$-valued invariant $s(M, g)$ (cf. 2.12) for positive scalar curvature metrics $g$ on closed $(4 k-1)$-dimensional spin manifolds $M$ with vanishing real Pontrjagin classes. This invariant is related to an integervalued invariant $i\left(g_{0}, g_{1}\right)$ defined by Gromov and Lawson [GL2, Definition 3.13] for pairs of positive scalar curvature metrics $g_{i}$ on a $(4 k-1)$-dimensional spin manifold. More precisely, if the real Pontrjagin classes of $M$ vanish then

$$
i\left(g_{0}, g_{1}\right)=s\left(M, g_{0}\right)-s\left(M, g_{1}\right) \text {. }
$$

These invariants are closely related to the Dirac operator on manifolds with boundary and hence we begin with a discussion of this operator and its index.

Let $W$ be a $4 k$-dimensional compact spin manifold with boundary $\partial W$. Let $g_{W}$ be a Riemannian metric which coincides with a product metric on $\partial W \times I$ in a collar neighbourhood of the boundary and let $g_{\partial W}$ be its restriction to $\partial W$. Let $D^{+}\left(W, g_{W}\right)$ be the (chiral) Dirac operator with respect to the metric $g_{W}$ from the positive to the negative spinors on $W$. This becomes a Fredholm operator if we impose the Atiyah-Singer-Patodi boundary condition, i.e. if we restrict to spinors on $W$ whose restriction to $\partial W$ is in the kernel of $P$, where $P$ is the spectral projection corresponding to eigenvalues $\geq 0$ of the (total) Dirac operator $D\left(\partial W, g_{\partial W}\right)$ on $\partial W$ [APS1, $\S \S 3$ and 4]. We denote by ind $D^{+}\left(W, g_{W}\right)$ the index of this Fredholm operator.

We recall that if $g_{W}(t)$ is a continuous family of metrics on $W$ then the corresponding family of spectral projections $P(t)$ is not continuous for those parameter values $t$ where an eigenvalue of $D\left(\partial W, g_{\partial W}(t)\right)$ crosses the origin. If $g_{\partial W}(t)$ has positive scalar curvature then Lichnerowicz's argument using the Weitzenböck formula shows that the kernel of $D\left(\partial W, g_{\partial W}(t)\right)$ is trivial [Li]. Hence $D^{+}\left(W, g_{W}(t)\right)$ is a continuous family of Fredholm operators and thus ind $D^{+}\left(W, g_{W}(t)\right)$ is independent of $t$. We note that if $g_{W}, g_{W}^{\prime}$ are two metrics on $W$ whose restrictions to the boundary are in the same component of $\mathfrak{R}_{\text {scal }}^{+}(\partial W)$ and which are product metrics near the boundary then they can 
be connected by a continuous family of such metrics. This implies the first of the following two remarks which we state for future reference.

Remark 2.2. (i) If $g_{W}$ is a metric on $W$ whose restriction to the boundary $g_{\partial W}$ has positive scalar curvature then ind $D^{+}\left(W, g_{W}\right)$ depends only on the connected component of $g_{\partial W}$ in $\mathfrak{R}_{\text {scal }}^{+}(\partial W)$. (ii) If $g_{W}$ has positive scalar curvature then ind $D^{+}\left(W, g_{W}\right)$ vanishes [APS2, p. 417].

The index theorem of Atiyah-Patodi-Singer [APS1, Theorem 4.2] gives the following formula for the index of $D^{+}\left(W, g_{W}\right)$ :

$$
\text { ind } D^{+}\left(W, g_{W}\right)=\int_{W} \hat{A}\left(p_{i}\left(W, g_{W}\right)\right)-\frac{h(\partial W)+\eta\left(D\left(\partial W, g_{\partial W}\right)\right)}{2} \text {. }
$$

Here $p_{i}\left(W, g_{W}\right)$ are the Pontrjagin forms of $W$ (with respect to the LeviCivita connection determined by $g_{W}$ ) and $\hat{A}$ is Hirzebruch's $\hat{A}$-polynomial. Moreover, $D\left(\partial W, g_{\partial W}\right)$ is the Dirac operator on $\partial W, h(\partial W)$ is the dimension of its kernel (which consists of the harmonic spinors on $\partial W$ ), and $\eta\left(D\left(\partial, g_{\partial W}\right)\right)$ is the $\eta$-invariant of Atiyah-Patodi-Singer, which measures the asymmetry of the spectrum of the selfadjoint operator $D\left(\partial W, g_{\partial W}\right)$.

Remark 2.4. If we glue $\left(W, g_{W}\right)$ and $\left(W^{\prime}, g_{W}^{\prime}\right)$ along isometric boundary components then the index formula shows that the index of the Dirac operator behaves additively, provided that there are no harmonic spinors on that common boundary component, e.g. if the scalar curvature on that piece of the boundary is positive.

Next we recall the definition of the Gromov-Lawson invariant $i\left(g_{0}, g_{1}\right)$ using a slightly different (but completely equivalent [APS1, Corollary 3.14]) formulation. Let $M$ be a closed spin manifold of dimension $4 k-1$ and let $g_{0}$ and $g_{1}$ be positive scalar curvature metrics on $M$. In this situation Gromov and Lawson [GL2, Definition 3.13] define an invariant $i\left(g_{0}, g_{1}\right) \in \mathbb{Z}$ by

$$
i\left(g_{0}, g_{1}\right)=\text { ind } D^{+}(M \times I, G),
$$

where $G$ is any metric on $M \times I$ which restricts to $g_{i}$ on $M \times i$ for $i=0,1$ and which is a product metric in a collar neighbourhood of the boundary. The first part of Remark 2.2 shows that $i\left(g_{0}, g_{1}\right)$ depends only on the components of $g_{0}, g_{1}$ in $\mathfrak{R}_{\text {scal }}^{+}(M)$. The second part implies that $i\left(g_{0}, g_{1}\right)$ vanishes if $g_{0}, g_{1}$ are in the same component (the path connecting $g_{0}$ and $g_{1}$ defines a metric $h$ on $M \times I$ which after shrinking in $M$-direction has positive scalar curvature).

We show below (cf. 2.16) that it is not possible to define an invariant $s(M, g)$ $\in \mathbb{Q}$ depending only on the spin isometry class of $(M, g)$ (a spin isometry is a spin structure preserving isometry) and satisfying (2.1) for all (4k-1)dimensional spin manifolds $M$. However, we define such an invariant for spin manifolds $M$ satisfying the following

Assumption 2.6. All real Pontrjagin classes of $M$ vanish.

The idea behind the definition of $s(M, g)$ is to rewrite the right-hand side of the index formula (2.3) as a sum of two terms, one depending only on the geom- 
etry of $\partial W$, the other depending only on the topology of $W$. Assuming that the real Pontrjagin classes of $\partial W$ vanish this can be done. The following lemma shows how the integral over the decomposable summands in $\int_{W} \hat{A}\left(p_{i}(W, g)\right)$ can be written as such a sum.

Lemma 2.7. Let $\alpha$ and $\beta$ be closed differential forms of positive degree on $W$ whose restrictions to $\partial W$ are coboundaries; i.e. there are forms $\hat{\alpha}, \hat{\beta}$ on $\partial W$ such that $d \hat{\alpha}=\alpha_{\mid \partial W}$ and $d \hat{\beta}=\beta_{\mid \partial W}$. Then

$$
\int_{W} \alpha \wedge \beta=\int_{\partial W} \hat{\alpha} \wedge \beta+\left\langle j^{-1}[\alpha] \cup j^{-1}[\beta],[W, \partial W]\right\rangle,
$$

where $j^{-1}[\alpha] \in H^{*}(W, \partial W ; \mathbb{R})$ is any preimage of the deRham cohomology class $[\alpha] \in H^{*}(W ; \mathbb{R})$ under the natural map $j: H^{*}(W, \partial W ; \mathbb{R}) \rightarrow H^{*}(W ; \mathbb{R})$, $j^{-1}[\beta]$ is defined analogously, and $\langle,[W, \partial W]\rangle$ is the Kronecker product with the fundamental class.

The proof is an easy consequence of Stokes's Theorem (compare [KS1, p. 380]). We note that the integral on the right-hand side is independent of the choice of $\hat{\alpha}$ and that the Kronecker product is independent of the choice of the preimages $j^{-1}[\alpha]$ and $j^{-1}[\beta]$. Slightly abusing language we define

$$
\int_{\partial W} d^{-1}(\alpha \wedge \beta):=\int_{\partial W} \hat{\alpha} \wedge \beta
$$

To get rid of the indecomposable summand in $\hat{A}\left(p_{i}(W, g)\right.$ ) (which is a nontrivial multiple of the Pontrjagin form $\left.p_{k}(W, g)\right)$ we observe that a suitable linear combination of the $\hat{A}$-polynomial and Hirzebruch's $L$-polynomial, namely $\hat{A}+a_{k} L$ with $a_{k}=1 /\left(2^{2 k+1}\left(2^{2 k-1}-1\right)\right)$, is in degrees $\leq 4 k$ a polynomial in the $p_{i}$ 's for $i<k$; i.e. it does not involve $p_{k}$ [Hir]. The signature theorem for manifolds with boundary [APS1, Theorem 4.14] gives

$$
\operatorname{sign}(W)=\int_{W} L\left(p_{i}(W, g)\right)-\eta\left(B\left(\partial W, g_{\mid \partial W}\right)\right),
$$

where $B$ is the signature operator. This can be interpreted as a formula expressing the integral as a sum of two terms, one involving only the geometry of the boundary, the other only the topology of $W$.

Assuming that the real Pontrjagin classes of $\partial W$ vanish and combining (2.3), (2.7), and (2.9) we obtain

$$
\text { ind } \begin{aligned}
D^{+}\left(W, g_{W}\right)= & \int_{\partial W} d^{-1}\left(\hat{A}+a_{k} L\right)\left(p_{i}\left(\partial W, g_{\mid \partial W}\right)\right) \\
& -\frac{h(\partial W)+\eta\left(D\left(\partial W, g_{\mid \partial W}\right)\right)}{2} \\
& -a_{k} \eta\left(B\left(\partial W, g_{\mid \partial W}\right)\right)-t(W),
\end{aligned}
$$

where the "topological" term $t(W)$ is given by

$$
t(W)=-\left\langle\left(\hat{A}+a_{k} L\right)\left(j^{-1} p_{i}(W)\right),[W, \partial W]\right\rangle+a_{k} \operatorname{sign}(W) .
$$


Here $p_{i}(W)$ denotes the $i$ th real Pontrjagin class of $W$ and $j^{-1} p_{i}(W)$ is any preimage under the natural map $j: H^{*}(W, \partial W ; \mathbb{R}) \rightarrow H^{*}(W ; \mathbb{R}$ ) (which exists due to the assumption that $p_{i}(\partial W)$ vanishes).

In particular, if all real Pontrjagin classes of $M$ vanish we can apply (2.10) to $W=M \times I$. In this case $t(W)$ vanishes and thus we define

Definition 2.12. Given a closed $(4 k-1)$-dimensional spin manifold $M$ with vanishing real Pontrjagin classes and positive scalar curvature metric $g$ on $M$ we define

$$
s(M, g)=-\frac{1}{2} \eta(D(M, g))-a_{k} \eta(B(M, g))+\int_{M} d^{-1}\left(\hat{A}+a_{k} L\right)\left(p_{i}(M, g)\right) .
$$

Next we summarize some basic properties of the $s$-invariant. If $M$ and $M^{\prime}$ are manifolds of dimension $\geq 4$ and $g, g^{\prime}$ are positive scalar curvature metrics on $M$ (resp. $M^{\prime}$ ), then there is a positive scalar curvature metric $g \# g^{\prime}$ on the connected sum $M \# M^{\prime}$ [GL1]. The metric $g \# g^{\prime}$ depends on some choices but it is not hard to see that different choices lead to metrics in the same connected component of $\mathfrak{R}_{\text {scal }}^{+}\left(M \# M^{\prime}\right)$.

Proposition 2.13. Let $M, M^{\prime}$ be $(4 k-1)$-dimensional closed spin manifolds with vanishing real Pontrjagin classes and let $g, g^{\prime}$ be positive scalar curvature metrics on $M$ (resp. $\left.M^{\prime}\right)$.

(i) If $f:(M, g) \rightarrow\left(M^{\prime}, g^{\prime}\right)$ is a spin isometry (i.e. a spin structure preserving isometry), then $s(M, g)=s\left(M^{\prime}, g^{\prime}\right)$.

(ii) $s(M, g)$ depends only on the connected component of $g$ in $\mathfrak{R}_{\text {scal }}^{+}(M)$.

(iii) If $M$ bounds a spin manifold $W$ and $g_{W}$ is a metric on $W$ extending $g$, which is a product metric near the boundary then

$$
s(M, g)=\operatorname{ind} D^{+}\left(W, g_{W}\right)+t(W) .
$$

(iv) $s\left(M \# M^{\prime}, g \# g^{\prime}\right)=s(M, g)+s\left(M^{\prime}, g^{\prime}\right)$.

Proof. The parts (i)-(iii) are immediate consequences of the definition of $s$ and the discussion above. For (iv) we note that the connected sum $M \# M^{\prime}$ is obtained from the disjoint union $M \amalg M^{\prime}$ by a 0 -surgery. Let $W$ denote the trace of this surgery, which is a bordism between $M \amalg M^{\prime}$ and $M \# M^{\prime}$ obtained by attaching a 0 -handle to $\left(M \amalg M^{\prime}\right) \times I$. By results of [Ga] or [Ca, Lemma 10] there is a positive scalar curvature metric $g_{W}$ on $W$ which restricts to $g$ (resp. $g^{\prime}$, resp. $g \# g^{\prime}$ ) on the boundary components of $W$ and is a product metric near the boundary. From 2.13 (iii) and 2.2 (ii) we conclude

$$
s\left(M \# M^{\prime}, g \# g^{\prime}\right)-s(M, g)-s\left(M^{\prime}, g^{\prime}\right)=\operatorname{ind} D^{+}\left(W, g_{W}\right)+t(W)=0,
$$

which proves part (iv).

Since the disjoint union of two copies of every $(4 k-1)$-dimensional spin manifold bounds [ABP] we can take 2.13 (iii) as a definition of $s(M, g)$. Also, 2.13 (iii) implies that $s(M, g)$ is a rational number. Reducing $s$ modulo $\mathbb{Z}$ (for $k$ even), respectively $2 \mathbb{Z}$ ( for $k$ odd) we get an invariant depending 
only on the diffeomorphism type of $M$ (considered as spin manifold), since ind $D^{+}\left(W, g_{W}\right)$ is an integer (resp. even integer for $k$ odd; $c f$. the remarks following Proposition 2.16). For $k=2$ this is (up to a sign) the invariant $s_{1}$ used in [KS1] and [KS2] for the diffeomorphism classification of certain 7-manifolds (cf. Theorems 3.4 and 3.9).

The invariant $s$ should be seen in analogy to invariants defined in [APS2], like the invariant $\tilde{\xi}_{\alpha}(0)$ [APS2, p.414]. As there, Definition 2.12 should be viewed as the fundamental one since it is intrinsically defined on $M$ whereas 2.13 (iii) involves an auxiliary manifold $W$. On the other hand the latter definition has the merit of being easier to compute as we will demonstrate in the next sections where we calculate the invariant for principal $S^{1}$-bundles with $S^{1}$-equivariant positive scalar curvature metric with totally geodesic fibres.

We will see in Theorem 3.11 that the $s$-invariant depends on the spin structure. To get an invariant of the moduli space we have to worry about diffeomorphisms which do not preserve the spin structure. We recall that after fixing an orientation of $M$ the spin structures compatible with that orientation are parametrized by $H^{1}(M ; \mathbb{Z} / 2)$. In particular, the spin structure is determined by the orientation if we assume that $H^{1}(M ; \mathbb{Z} / 2)$ vanishes. If we change the orientation, the $s$-invariant changes its sign. Summarizing we conclude:

Proposition 2.14. If $M$ is a closed connected spin manifold of dimension $4 k-1$ with vanishing real Pontrjagin classes and $H^{1}(M ; \mathbb{Z} / 2)=0$, then $s$ induces $a$ map

$$
|s|: \pi_{0}\left(\mathfrak{R}_{\text {scal }}^{+}(M) / \operatorname{Diff}(M)\right) \rightarrow \mathbb{Q} .
$$

Corollary 2.15. Let $M$ be a closed connected spin manifold of dimension $4 k-1$, for $k>1$, with vanishing real Pontrjagin classes and $H^{1}(M ; \mathbb{Z} / 2)=0$. If $\mathfrak{R}_{\mathrm{scal}}^{+}(M)$ is not empty (which is the case for a simply connected $M$ by [St]), then the moduli space $\mathfrak{R}_{\text {scal }}^{+}(M) / \operatorname{Diff}(M)$ has infinitely many connected components.

Proof. By the proposition it suffices to construct infinitely many positive scalar curvature metrics on $M$ whose $|s|$-invariants are mutually different. Below we show that for $k>1$ there is an "exotic" positive scalar curvature metric $e$ on $S^{4 k-1}$ with $s\left(S^{4 k-1}, e\right)=1$ (resp. 2) for $k$ even (resp. odd). We note that the standard metric on $S^{4 k-1}$ has vanishing $s$-invariant since it extends to a positive scalar curvature metric on the $4 k$-disk. Then by 2.13 (iv) the connected sum of a fixed metric on $M$ and a number of copies of $e$ gives an infinite family of metrics on $M \# S^{4 k-1} \# \ldots \# S^{4 k-1}=M$ with different $s$-invariant.

The construction of $e$ is due to Carr [Ca, Proof of Theorem 4]. For the convenience of the reader and in order to calculate its $s$-invariant we recall the construction of $e$. Let $W$ be a closed $4 k$-dimensional spin manifold with $\hat{A}(W)=1$ (resp. 2 ) for $k$ even (resp. $k$ odd). Such a manifold can be constructed for example by plumbing as in [Ca] and hence we can assume that $W$ has a handle decomposition consisting of one 0 -handle and one $4 k$-handle and a number of $2 k$-handles. We decompose $W$ in the form $W=W_{-} \cup_{S^{4 k-1}}$ $W_{+}$, where $W_{-}$consists of the 0 -handle and the $2 k$-handles and $W_{+}$consists 
of the $4 k$-handle. We pick a positive scalar curvature metric on the 0 -handle which is a product near the boundary. According to [Ga] or [Ca, Lemma 10] this metric can be extended over the $2 k$-handles to give a positive scalar curvature metric on $W_{-}$which is a product metric near the boundary. Finally, this can be extended to a metric $g_{W}$ on all of $W$ (we are not asserting that $g_{W}$ has positive scalar curvature). Let $e$ be the restriction of $g_{W}$ to $S^{4 k-1}=W_{-} \cap W_{+}$. Then

$$
\begin{aligned}
s\left(S^{4 k-1}, e\right) & =\operatorname{ind} D^{+}\left(W_{+}, g_{W_{+}}\right)+t\left(W_{+}\right) \\
& =\operatorname{ind} D^{+}\left(W_{+}, g_{W_{+}}\right)+\operatorname{ind} D^{+}\left(W_{-}, g_{W_{-}}\right) \\
& =\operatorname{ind} D^{+}\left(W, g_{W}\right)=\hat{A}(W) .
\end{aligned}
$$

Here $g_{W_{ \pm}}$denotes the restriction of $g_{W}$ to $W_{ \pm}$. The second equality holds since $t\left(W_{+}\right)$vanishes (this follows directly from the definition of $t$ (2.11) since $W_{+}$is a disk) and ind $D^{+}\left(W_{-}, g_{W}\right)=0$ by 2.2 (ii) since $g_{W}$ has positive scalar curvature. The third equality follows from the additivity of the index (cf. 2.4).

We end this section by showing that an invariant $s(M, g)$ with the property (2.1) depending only on the spin isometry class of $(M, g)$ cannot be defined without extra assumptions on $M$. Suppose $M$ is a $(4 k-1)$-dimensional spin manifold for which we can define a $\mathbb{Q}$-valued invariant $s(M, g)$ with the property (2.1) for all positive scalar curvature metrics on $M$. If $f: M \rightarrow M$ is a spin structure preserving self diffeomorphism then $i\left(g, f^{*} g\right)=s(M, g)-$ $s\left(M, f^{*} g\right)=0$, since $f$ is a spin isometry from $\left(M, f^{*} g\right)$ to $(M, g)$ and hence their $s$-invariants agree.

Gromov and Lawson show that the map

$$
i_{g}: \operatorname{Diff}(M) \rightarrow \mathbb{Z}
$$

defined by $i_{g}(f)=i\left(g, f^{*} g\right)$ is a group homomorphism which factors through the component group $\pi_{0}(\operatorname{Diff}(M))$ of the diffeomorphism group of $M$ [GL2, 4.48 and 4.49].

Proposition 2.16. For $k>1$ there is a closed spin manifold $M$ of dimension $4 k-1$ and a positive scalar curvature metric $g$ on $M$ such that the image of $i_{g}$ restricted to the spin structure preserving diffeomorphisms is $\mathbb{Z}$ for $k$ even and $2 \mathbb{Z}$ for $k$ odd.

We remark that $i_{g}(f)$ for $k$ odd is always an even integer since the space of spinors on a $4 k$-dimensional spin manifold $W$ has the structure of a quaternionic vector space and since the Dirac operator as well as the Atiyah-PatodiSinger boundary condition is $\mathbb{H}$-linear.

Proof. Recall that $i\left(g, f^{*} g\right)=$ ind $D^{+}(M \times I, G)$, where $G$ is a metric on $M \times I$ which restricts to $g$ (resp. $f^{*} g$ ) on the boundary and is a product metric in a collar neighbourhood. Now we apply the index theorem (2.3) and note that $h=0$ (since the metric on the boundary has positive scalar curvature) 
and that the $\eta$-invariant is zero, provided that $f$ is spin structure preserving (since the $\eta$-invariants of both boundary components are the same up to a minus sign). Hence if we assume that $f$ is spin structure preserving then

$$
\begin{aligned}
i\left(g, f^{*} g\right) & =\operatorname{ind} D^{+}(M \times I, G)=\int_{M \times I} \hat{A}\left(p_{i}(M \times I), G\right) \\
& =\int_{M_{f}} \hat{A}\left(p_{i}\left(M_{f}\right), G\right)=\hat{A}\left(M_{f}\right),
\end{aligned}
$$

where $M_{f}$ is the mapping torus of $f$ (obtained by identifying in $M \times I$ every point $(x, 1)$ with $(f(x), 0))$.

We recall that for $k \geq 1$ there is a spin manifold $N$ of dimension $4 k$ with $\hat{A}(N)=1$ for $k$ even and $\hat{A}(N)=2$ for $k$ odd. Replacing $N$ for $k>1$ by the disjoint union of $N$ and copies of $\mathbb{H P}^{2 l}$ (for $k=2 l$ ) or $\mathbb{H}^{2 l} \times K$ (for $k=2 l+1$ ) we can also assume that the signature of $N$ vanishes. Here $\mathbb{H}^{2 l}$ is the quaternionic projective space with sign $\mathbb{H} \mathbb{P}^{2 l}=1$ and $K$ is the Kummer surface, a 4-dimensional spin manifold with signature 16 (recall that the signature of $(8 l+4)$-dimensional spin manifolds is divisible by 16 [Oc] and that $\hat{A}\left(\mathbb{H P}^{2 l}\right)=0[\mathrm{BH}]$, a fact which also follows since the standard metric on $\mathbb{H}^{2 l}$ has positive scalar curvature). A result of the first author implies that $N$ is spin bordant to the mapping torus $M_{f}$ of a spin structure preserving diffeomorphism on a zero bordant spin manifold $M$ [Kr, Theorem 9.9]. The proof of the result shows that we can choose $M$ to be simply connected. This implies that $M$ carries a positive scalar curvature metric $g$ [GL1, Theorem B] and finishes the proof of the proposition.

\section{The EXAMPLeS}

In this section we discuss the families of positive sectional (resp. Ricci) curvature metrics on 7-manifolds constructed by Aloff and Wallach (resp. Wang and Ziller). Then we use the $s$-invariant in conjunction with results from [KS1, KS2] to find manifolds $M$ in these families for which $\mathfrak{R}_{\text {sec }}^{+}(M) / \operatorname{Diff}(M)$ is not connected, respectively where $\mathfrak{R}_{\text {Ric }}^{+}(M) / \operatorname{Diff}(M)$ has infinitely many components. We start with the discussion of the Wang-Ziller family.

The underlying manifolds are certain homogeneous spaces of the form $G / H$, where $G=S U(3) \times S U(2) \times U(1)$ and $H \cong S U(2) \times U(1) \times U(1)$, which were considered by Witten [Wi]. Equivalently they can be described as follows. For integers $k, l$ let $M_{k, l}$ be the principal $S^{1}$-bundle over $\mathbb{C P}^{2} \times \mathbb{C P}^{1}$ classified by $l x+k y \in H^{2}\left(\mathbb{C P}^{2} \times \mathbb{C P}^{1} ; \mathbb{Z}\right)$, where $x$ and $y$ are the generators of $H^{2}\left(\mathbb{C P}^{2} ; \mathbb{Z}\right)$, resp. $H^{2}\left(\mathbb{C P}^{1} ; \mathbb{Z}\right)$.

Theorem 3.1 (Wang-Ziller [WZ]). $M_{k, l}$ admits an Einstein metric $g_{k, l}$ with positive Einstein constant.

Their construction is the following. Let $\theta$ be the unique connection on $M_{k, l}$ whose curvature is the harmonic form representing $l x+k y$ (with respect to the standard metric on $\mathbb{C P}^{2} \times \mathbb{C P}^{1}$ ). For positive real numbers $x_{1}, x_{2}$ let $g$ 
be the metric on $\mathbb{C P}^{2} \times \mathbb{C P}^{1}$ obtained from the standard metric by rescaling by $x_{1}$ in the $\mathbb{C P}^{2}$-direction and $x_{2}$ in $\mathbb{C P}^{1}$-direction. Let $g_{k, l}$ be the $S^{1}$ equivariant metric on $M_{k, l}$ with totally geodesic fibres determined by $\theta, g$, and the standard metric on the fibres (cf. 4.3). Wang and Ziller show that for $x_{1}$, $x_{2}$ chosen suitably $g_{k, l}$ is an Einstein metric with positive Einstein constant and hence in particular $g_{k, l}$ is a metric with positive Ricci curvature. Since there might be more then one tuple $\left(x_{1}, x_{2}\right)$ such that the corresponding metric satisfies the Einstein equation, the metric $g_{k, l}$ might not be determined by $k$, l. But we observe that the component of $g_{k, l}$ in $\mathfrak{R}_{\text {scal }}^{+}\left(M_{k, l}\right)$ is determined by $k$ and $l$ since independent of $\left(x_{1}, x_{2}\right)$ the metric $g$ has positive scalar curvature and hence so does $g_{k, l}$ after rescaling the fibres if necessary (cf. remarks following 4.4).

We recall that $M_{k, l}$ is simply connected if $k$ and $l$ are coprime and admits a spin structure if $k$ is even. Moreover, $H^{4}\left(M_{k, l} ; \mathbb{Z}\right)$ is a finite cyclic group of order $l^{2}$. In particular, the real Pontrjagin classes of $M_{k, l}$ vanish. The following theorem is a consequence of our main technical result Theorem 3.11 below and the calculations in [KS1, §4].

Theorem 3.2. Let $k, l$ be coprime integers with $k$ even. Then

$$
s\left(M_{k, l}, g_{k, l}\right)=-\frac{3 k\left(l^{2}+3\right)\left(l^{2}-1\right)}{2^{7} 7 l^{2}} \text {. }
$$

Corollary 3.3. Let $(M, g)$ and $\left(M^{\prime}, g^{\prime}\right)$ be the total spaces of $S^{1}$-bundles over $\mathbb{C P}^{2} \times \mathbb{C P}^{1}$ given by coprime numbers $(k, l)$, resp. $\left(k^{\prime}, l^{\prime}\right)$, with $k$ and $k^{\prime}$ even. Then the following are equivalent:

(1) There is an orientation preserving diffeomorphism $f: M \rightarrow M^{\prime}$ such that $g$ and $f^{*} g^{\prime}$ are in the same component of $\mathfrak{R}_{\mathrm{scal}}^{+}(M)$.

(2) $l^{\prime}= \pm l$ and $k^{\prime}=k$.

Proof. (1) implies (2) since the order of $H^{4}(M, \mathbb{Z})$ determines $l$ up to sign and then $s(M, g)$ determines $k$. (2) implies (1) since complex conjugation on $\mathbb{C P}^{2}$ induces a fibre and orientation preserving isometry $\left(M_{k, l}, g_{k, l}\right) \rightarrow$ $\left(M_{k,-l}, g_{k,-l}\right)$.

Now we compare Corollary 3.3 with the diffeomorphism classification of the $M_{k, l}$ 's.

Theorem 3.4 [KS1]. Let $(k, l)$ and $\left(k^{\prime}, l^{\prime}\right)$ pairs of coprime integers with $k$, $k^{\prime}$ even. Then $M_{k, l}$ is orientation preserving diffeomorphic to $M_{k^{\prime}, l^{\prime}}$ if and only if $l^{\prime}= \pm l$ and $k^{\prime} \equiv k \bmod 2^{\lambda_{2}(l)} \cdot 7^{\lambda_{7}(l)} \cdot l^{2}$, where

$$
\lambda_{2}(l)=\left\{\begin{array}{l}
0 \text { for } l=2,6 \bmod 8, \\
1 \text { for } l=1,7 \bmod 8, \\
2 \text { for } l=3,5 \bmod 8, \\
3 \text { for } l=0,4 \bmod 8,
\end{array} \quad \lambda_{2}(l)=\left\{\begin{array}{l}
0 \quad \text { for } l=1,2,5,6 \bmod 7, \\
1 \quad \text { for } l=0,3,4 \bmod 7 .
\end{array}\right.\right.
$$


Combining Corollary 3.3 and Theorem 3.4 we see that for $l \neq 0,1$ leaving $l$ unchanged and adding multiples of $2^{\lambda_{2}(l)} \cdot 7^{\lambda_{7}(l)} \cdot l^{2}$ to $k$ give infinitely many diffeomorphic Einstein manifolds with positive Einstein constant and different $|s|$ invariant. Hence each $M=M_{k, l}$ with $k$ even and $l \neq 0,1$ carries infinitely many Einstein metrics which lie in different components of $\mathfrak{R}_{\text {scal }}^{+}(M) / \operatorname{Diff}(M)$. This proves Theorem 1.2.

Next we discuss the Aloff-Wallach family of positive sectional curvature manifolds. Let $k$ and $l$ be coprime integers and let

$$
i_{k, l}: S^{1} \rightarrow S U(3)
$$

be the inclusion which maps $z \in S^{1}$ to the diagonal matrix with entries $z^{k}, z^{l}$, $z^{-(k+l)}$. The Wallach spaces are the homogeneous spaces

$$
N_{k, l}=S U(3) / i_{(k, l)}\left(S^{1}\right) .
$$

These manifolds are simply connected. Let $g_{k, l}$ be the normal homogeneous metric on $N_{k, l}$, which is unique modulo scaling (the normal homogeneous metric on $N_{k, l}$ with respect to a bi-invariant metric on $S U(3)$ is defined by the property that it makes the projection map $S U(3) \rightarrow N_{k, l}$ a Riemannian submersion).

The normal homogeneous metric has positive scalar curvature, but the sectional curvature vanishes in certain tangent planes. Aloff and Wallach showed that one can get a metric with positive sectional curvature as follows. Consider $N_{k, l}$ as the total space of the bundle

$$
U(2) / i_{k, l}\left(S^{1}\right) \rightarrow S U(3) / i_{k, l}\left(S^{1}\right) \rightarrow S U(3) / U(2),
$$

where $U(2)$ is embedded in $S U(3)$ by mapping $A \in U(2)$ to the block matrix with $A$ in the upper left corner, $(\operatorname{det} A)^{-1}$ in the lower right corner, and zeros everywhere else. Aloff and Wallach show that rescaling the metric $g_{k, l}$ in vertical directions (with respect to (3.5)) by some $0<t<1$ one gets a homogeneous metric of positive sectional curvature, provided that $k l(k+l) \neq 0$ and $k l>0$ [AW, 3.1]. We call any such metric an Aloff-Wallach metric. Since for $t=0$ we get the normal homogeneous metric each Aloff-Wallach metric is in the same connected component of $\mathfrak{R}_{\text {scal }}^{+}\left(N_{k, l}\right)$ as the normal homogeneous metric. We observe that permuting $k, l$, and $-(k+l)$ leads to embeddings $S^{1} \rightarrow S U(3)$ conjugate to $i_{k, l}$ and hence to homogeneous spaces isometric to $\left(N_{k, l}, g_{k, l}\right)$. Doing such permutations if necessary we can always assume $k l>0$ and hence we can formulate the result of Aloff and Wallach as follows.

Theorem 3.6 (Aloff-Wallach [AW]). If $k l(k+l) \neq 0$, the Aloff-Wallach metrics on $N_{(k, l)}$ have positive sectional curvature. They are in the same connected component of the space of positive scalar curvature metrics as the normal homogeneous metric $g_{k, l}$.

Now we want to compare the isometry classification of the $N_{k, l}$ 's with their diffeomorphism classification. As noted above permuting $k, l$, and $-(k+l)$ 
leads to conjugate embeddings. The simplest numerical functions of $k$ and $l$ which are invariant under these substitutions are

$$
N(k, l)=k^{2}+k l+l^{2} \quad \text { and } \quad T(k, l)=k l(k+l) .
$$

Observing that the Weyl group of $S U(3)$ is the permutation group of three letters we conclude that $i_{k, l}$ is conjugate to $i_{k^{\prime}, l^{\prime}}$ if and only if $N(k, l)=$ $N\left(k^{\prime}, l^{\prime}\right)$ and $T(k, l)=T\left(k^{\prime}, l^{\prime}\right)$. We recall that $N(k, l)$ is the order of $H^{4}\left(N_{k, l} ; \mathbb{Z}\right)[\mathrm{AW}]$.

The following calculation of the $s$-invariant follows again from our main technical result Theorem 3.11 and computations in [KS2,§4].

Theorem 3.7. For any Aloff-Wallach metric $g$

$$
s\left(N_{k, l}, g\right)=s\left(N_{k, l}, g_{k, l}\right)=\frac{1}{2^{5} 7} T(k, l),
$$

where $g_{k, l}$ is the normal homogeneous metric.

Corollary 3.8. Let $(k, l)$, resp. $\left(k^{\prime}, l^{\prime}\right)$, be pairs of coprime numbers and $(N, g)$ resp. $\left(N^{\prime}, g^{\prime}\right)$, the Wallach space together with its normal homogeneous metric determined by $(k, l)$, resp. $\left(k^{\prime}, l^{\prime}\right)$. Then the following are equivalent:

(1) There is an orientation preserving diffeomorphism $f: N \rightarrow N^{\prime}$ such that $g$ and $f^{*} g^{\prime}$ are in the same component of $\mathfrak{R}_{\text {scal }}^{+}(N)$.

(2) The subgroups $i_{k, l}\left(S^{1}\right)$ and $i_{k^{\prime}, l^{\prime}}\left(S^{1}\right)$ are conjugate in $S U(3)$.

(3) $N(k, l)=N\left(k^{\prime}, l^{\prime}\right)$ and $T(k, l)=T\left(k^{\prime}, l^{\prime}\right)$.

Next we recall the diffeomorphism classification of the Wallach spaces.

Theorem 3.9 [KS2, p. 466]. Two Wallach spaces $N_{k, l}$ and $N_{k^{\prime}, l^{\prime}}$ are orientation preserving diffeomorphic if and only if

$$
N(k, l)=N\left(k^{\prime}, l^{\prime}\right) \text { and } T(k, l) \equiv T\left(k^{\prime}, l^{\prime}\right) \bmod 2^{5} \cdot 3 \cdot 7^{\lambda(N)} \cdot N,
$$

where $N=N(k, l)$ and $\lambda(N)=0$ if $N \equiv 0 \bmod 7$ and $\lambda(N)=1$ otherwise.

To finish the proof of our main result Theorem 1.1 we want to find diffeomorphic Wallach spaces with different $|s|$-invariants. This is not easy since the corresponding number theory is ugly and not understood. By a computer search Don Zagier and Andrew Odlyzko found this way three solutions [KS2, p. 479]. The smallest one (in the sense that $H^{4}$ is minimal) is the following:

Example 3.10. $N_{-4638661,582656}$ is diffeomorphic to $N_{-2594149,5052965}$ but for any Aloff-Wallach metric the $|s|$-invariants are different.

The order of $H^{4}$ is 19153920223641 . The order in the two other known cases are 411358875444559 and 2738810764243641 . This finishes the proof of Theorem 1.1.

To prove Theorems 3.2 and 3.7 we note that both families considered above are total spaces of principal $S^{1}$-bundles and that the Wang-Ziller metrics as well as the normal homogeneous metric on $S U(3) / S^{1}$ are $S^{1}$-equivariant metrics 
with totally geodesic fibres. Hence we will derive below a general formula for the $s$-invariant of such a positive scalar curvature metric $g$ on the total space of a principal $S^{1}$-bundle $p: P \rightarrow B$. It turns out that the answer depends on which spin structure we choose on $P$ and so we begin with a discussion of these choices.

Note that the tangent bundle $T P$ is isomorphic to $p^{*}(T B) \oplus T_{F} P$ and that the tangent bundle along the fibres $T_{F} P$ is trivial (the vector field generating the $S^{1}$-action on $P$ provides a trivialization of $T_{F} P$ ). Hence a spin structure on $B$ (which exists if the second Stiefel Whitney class $w_{2}(B)$ vanishes) induces a spin structure on $B$ which we denote by $\phi$.

If $w_{2}(B)=c \bmod 2$, where $c=c_{1}(E)$ is the first Chern class of the complex line bundle $E$ associated to $p: P \rightarrow B$, then $T B \oplus E$ admits a spin structure. The choice of such a spin structure gives a spin structure on the disk bundle $D E$ whose restriction to the sphere bundle $S E=P$ we denote by $\phi^{\prime}$. Note that if $w_{2}(B)=0$ and $c=0 \bmod 2$, then $\phi$ and $\phi^{\prime}$ are different spin structures on $P$, since the restriction of $\phi$ to a fibre $S^{1}$ is the nontrivial spin structure, which does not extend over $D^{2}$, whereas the restriction of $\phi^{\prime}$ extends by construction.

Recall that our invariant $s(P, g)$ is only defined if the real Pontrjagin classes $p_{i}(P) \in H^{4 i}(P ; \mathbb{R})$ vanish. The isomorphism $T P \cong p^{*}(T B) \oplus T_{F} P$ and the triviality of $T_{F} P$ imply $p^{*}\left(p_{i}(B)\right)=p_{i}(P)=0$ and hence the Gysin exact sequence

$$
\rightarrow H^{4 i-2}(B ; \mathbb{R}) \stackrel{\cup c}{\longrightarrow} H^{4 i}(B ; \mathbb{R}) \stackrel{p^{*}}{\longrightarrow} H^{4 i}(P ; \mathbb{R}) \longrightarrow
$$

shows that $p_{i}(B)$ is divisible by $c$; i.e. there are elements $\bar{p}_{i} \in H^{4 i-2}(B ; \mathbb{R})$ such that $p_{i}(B)=\bar{p}_{i} c$.

Theorem 3.11. Let $p: P \rightarrow B$ be a principal $S^{1}$-bundle over a closed oriented manifold $B$ of dimension $4 k-2$. Let $c \in H^{2}(B ; \mathbb{Z})$ be the first Chern class of the associated complex line bundle. Let $g$ be a $S^{1}$-equivariant positive scalar curvature metric on $P$ with totally geodesic fibres and let $\phi, \phi^{\prime}$ be the spin structures on $P$ described above (which exist if $w_{2}(B)=0$, resp. if $w_{2}(B)=c$ mod 2). Assume that the real Pontrjagin classes $p_{i}(P) \in H^{4 i}(P ; \mathbb{R})$ vanish (so that $s(P, g)$ is defined $)$. Then

$$
\begin{aligned}
& s(P, \phi, g)=-\left\langle\hat{A}(T B) \frac{1}{2 \tanh (c / 2)}+a_{k} L(T B) \frac{1}{\tanh c},[B]\right\rangle+a_{k} \operatorname{sign}\left(B_{c}\right), \\
& s\left(P, \phi^{\prime}, g\right)=\left\langle\hat{A}(T B) \frac{1}{2 \sinh (c / 2)}+a_{k} L(T B) \frac{1}{\tanh c},[B]\right\rangle+a_{k} \operatorname{sign}\left(B_{c}\right) .
\end{aligned}
$$

Here $\hat{A}(T B)$ (resp. $L(T B)$ ) are the usual polynomials in the Pontrjagin classes of $B, c$ is the first Chern class of $E, a_{k}=1 /\left(2^{2 k+1}\left(2^{2 k-1}-1\right)\right),\langle,[B]\rangle$ is the evaluation on the fundamental class of $B$, and $\operatorname{sign}\left(B_{c}\right)$ is the signature of the bilinear form $B_{c}: H^{2 k-2}(B) \otimes H^{2 k-2}(B) \rightarrow \mathbb{R}$ defined by $B_{c}(x \otimes y)=$ $\langle x y c,[B]\rangle$.

We remark that the expressions $\frac{1}{2 \sinh (c / 2)}, \frac{1}{2 \tanh (c / 2)}$, and $\frac{1}{\tanh c}$ in the above formula have to be interpreted as (Laurent) series of the form $1 / c+k_{0}+k_{1} c+$ 
$k_{2} c^{2}+\cdots$, where the coefficients $k_{i}$ can be expressed in terms of Bernoulli numbers (cf. [MS, Appendix B]). The number $a_{k}$ is chosen such that the linear combination $\hat{A}+a_{k} L$ in degrees $\leq 4 k$ is a polynomial in the Pontrjagin classes $p_{i}(B)$ for $i<k$. Recall that the Pontrjagin classes $p_{i}(B)$ can be written in the form $p_{i}(B)=\bar{p}_{i} c$ and hence the expressions $\hat{A}(T B) \frac{1}{2 \tanh (c / 2)}+a_{k} L(T B) \frac{1}{\tanh c}$ and $\hat{A}(T B) \frac{1}{2 \sinh (c / 2)}+a_{k} L(T B) \frac{1}{\tanh c}$ are polynomials. For example, for $k=2$ we get the following explicit formulas:

$$
\begin{aligned}
& s(P, \phi, g)=\frac{1}{2^{7} \cdot 3 \cdot 7}\left\langle-3 p_{1}(B) \bar{p}_{1}+8 p_{1}(B) c+4 c^{3},[B]\right\rangle+\frac{1}{2^{5} \cdot 7} \operatorname{sign}\left(B_{c}\right), \\
& s\left(P, \phi^{\prime}, g\right)=\frac{1}{2^{7} \cdot 7}\left\langle-p_{1}(B) \bar{p}_{1}-2 p_{1}(B) c-c^{3},[B]\right\rangle+\frac{1}{2^{5} \cdot 7} \operatorname{sign}\left(B_{c}\right) .
\end{aligned}
$$

\section{THE $S$-INVARIANT FOR $S^{1}$-BUNDLES}

The goal of this section and the next section is to provide the proof of Theorem 3.11, i.e. to calculate $s(P, g)$ where $P$ is the total space of a principal $S^{1}$-bundle $p: P \rightarrow B$ over a closed oriented manifold $B$ of dimension $4 k-2$ and $g$ is a $S^{1}$-equivariant metric with totally geodesic fibres. Recall that the invariant $s(P, g)$ can be determined alternatively by using Definition 2.12 calculating integrals and $\eta$-invariants on $P$, or by using formula 2.13 (iii) calculating characteristic numbers and the index of the Dirac operator on a spin manifold $W$ which bounds $P$ :

$$
s(P, g)=\operatorname{ind} D^{+}\left(W, g_{W}\right)+t(W)
$$

where $t(W)=-\left\langle\left(\hat{A}+a_{k} L\right)\left(j^{-1} p_{i}(W)\right),[W, \partial W]\right\rangle+a_{k} \operatorname{sign}(W)$. Here $D^{+}\left(W, g_{W}\right)$ is the Dirac operator on $W$ with respect to a metric $g_{W}$ on $W$ which restricts to $g$ on the boundary and is the product metric in a collar neighbourhood of the boundary. Recall that the index of $D^{+}\left(W, g_{W}\right)$ depends only on the connected component of the positive scalar curvature metric $g$. In general it is very difficult to compute ind $D^{+}\left(W, g_{W}\right)$, but if $g_{W}$ has positive scalar curvature then ind $D^{+}\left(W, g_{W}\right)=0$ (cf. 2.2 (ii)).

In our situation there is an obvious manifold with boundary $P$, namely the disk bundle $D E$. Recall from $\S 3$ that $D E$ is a spin manifold if and only if $w_{2}(B)=c \bmod 2$ and that in that case the induced spin structure on $\partial D E=P$ is $\phi^{\prime}$.

Lemma 4.2. (1) If $w_{2}(B)=c$ mod 2 then ind $D^{+}\left(D E, g_{D E}\right)=0$ for any metric $g_{D E}$ on $D E$ which restricts to $g$ on the boundary and is a product metric in a collar neighbourhood of the boundary.

(2) $t(D E)=-\left\langle\hat{A}(T B) \frac{1}{2 \sinh (c / 2)}+a_{k} L(T B) \frac{1}{\tanh c},[B]\right\rangle+a_{k} \operatorname{sign}\left(B_{c}\right)$.

Proof. For the first part it suffices to construct a positive scalar curvature metric $g_{D E}$ on $D E$ which restricts to $g$ on the boundary (or to a positive scalar curvature metric in the same connected component as $g$ ) and is a product metric in a collar neighbourhood of the boundary. For this it is convenient to 
consider $D E$ as the total space of a $D^{2}$-bundle associated to the $S^{1}$-principal bundle $P \rightarrow B$, and to use the following result of Vilms.

Proposition 4.3 (cf. [Be, 9.59]). Let $G$ be a compact Lie group and let $F$ be a manifold with a smooth $G$-action. Let $P \rightarrow B$ be a principal $G$-bundle and let $\pi: M=P \times_{G} F \rightarrow B$ be the associated bundle with fibre $F$. Given a Riemannian metric $g_{B}$ on $B, a$-invariant Riemannian metric $g_{F}$ on $F$, and a principal connection $\theta$ on $P$, there exists one and only one Riemannian metric $g$ on $M$ such that $\pi$ is a Riemannian submersion from $\left(M, g_{M}\right)$ to $\left(B, g_{B}\right)$ with totally geodesic fibres isometric to $\left(F, g_{F}\right)$ and horizontal distribution associated to $\theta$.

Rescaling $g$ in fibre direction, i.e. replacing $g_{F}$ by $t g_{F}$ for some $t>0$, we get a family of Riemannian metrics $g_{t}$ on $M$. The scalar curvature $s_{t}$ of this family is given by [Be, 9.70]

$$
s_{t}=\frac{1}{t} s_{F}+s_{B} \circ \pi-t|A|^{2}
$$

where $s_{F}, s_{B}$ is the scalar curvature of $F$, resp. $B$, and $A$ is a tensor defined by O'Neill (essentially the curvature of $\theta$ [Be, 9.54c]). It follows that for $s_{F} \geq 0$ and $s_{B}>0$, decreasing $t$ increases the scalar curvature of $g_{t}$ and for sufficiently small $t$ the scalar curvature of $g_{t}$ is positive. In particular, in this case the connected component of $g_{t} \in \mathfrak{R}_{\text {scal }}^{+}(M)$ depends only on $g_{B}$, not on $\theta$ or the scaling $t$ (provided that $t$ is small enough to make the scalar curvature of $g_{t}$ positive).

Now we specialize to the situation where $P \rightarrow B$ is our principal $S^{1}$-bundle. Let $g_{B}$ be the metric on $B$ and let $\theta$ be the connection on $P$ determined by the metric $g$. The scalar curvature of $g_{B}$ is positive by (4.4) since the scalar curvature of the fibres is zero. Let $g_{D^{2}}$ be a $S^{1}$-equivariant metric on the disk $D^{2}$ which has nonnegative scalar curvature, restricts to the standard metric on the boundary, and is a product near the boundary. We note that the induced metric of $D^{2}$ when considered as a hemisphere in $S^{2}$ has all these properties except the last one, but this can be achieved as follows. In polar coordinates around the north pole the standard metric on $S^{2}$ has the form $d r^{2}+\sin ^{2} r d \phi^{2}$. Now replace $\sin r$ by a function $f(r)$ with $f^{\prime \prime} \geq 0$ which agrees with $\sin r$ for small $r$ and is identically equal to 1 for $r$ close to $\pi / 2$. The resulting metric on the upper hemisphere has the required properties.

Let $g_{t}$ be the family of metrics on the associated bundle $D E=P \times_{S^{1}} D^{2}$ determined by $g_{B}, \theta$, and $t g_{D^{2}}$ via (4.3). The metric $g_{t}$ restricts to a product metric in a collar neighbourhood of the boundary. For sufficiently small $t$ the metric $g_{t}$ has positive scalar curvature by (4.4) and hence ind $D^{+}\left(D E, g_{t}\right)=0$ by 2.2 (ii). Restricted to the boundary $g_{t}$ agrees with the original metric $g$ rescaled in fibre direction. Again by (4.4) the metric $g$ and this rescaled metric are in the same connected component of $\mathfrak{R}_{\text {scal }}^{+}(P)$. By 2.2 (i) this implies the first part of Lemma 4.2.

For the proof of the second part recall from the definition of the $\hat{A}$-class, resp. the $L$-class [Hir], that for a complex line bundle $E$ with first Chern class 
$c$ we have

$$
\hat{A}(E)=\frac{c}{2 \sinh (c / 2)} \quad \text { and } \quad L(E)=\frac{c}{\tanh c} .
$$

To evaluate $\left(\hat{A}+a_{k} L\right)\left(j^{-1} p_{i}(D E)\right)$ we note that $j: H^{*}(D E, \partial) \rightarrow H^{*}(D E) \cong$ $H^{*}(B)$ maps the Thom class $U \in H^{2}(D E, \partial)$ to $c$ and hence $\bar{p}_{i} U$ to $\bar{p}_{i} c=$ $p_{i}(B)$. Using the bundle equation $T D E=p^{*}(T B \oplus E)$ we get

$$
\left(\hat{A}+a_{k} L\right)\left(j^{-1} p_{i}(D E)\right)=\hat{A}(T B) \frac{U}{2 \sinh (U / 2)}+a_{k} L(T B) \frac{U}{\tanh U},
$$

which by the remarks following Theorem 3.11 can be written in the form $q\left(\bar{p}_{i}, U\right) \cup U$, where $q\left(\bar{p}_{i}, U\right)$ is a polynomial in the $\bar{p}_{i}$ 's, $i<k$, and $U$. It follows that

$$
t(D E)=\left\langle q\left(\bar{p}_{i}, U\right) \cup U,[D E, \partial]\right\rangle=\left\langle q\left(\bar{p}_{i}, c\right), U \cap[D E, \partial]\right\rangle=\left\langle q\left(\bar{p}_{i}, c\right),[B]\right\rangle .
$$

Finally we note that the cup product form on $H^{2 k}(D E, \partial D E)$ corresponds to the form $B_{c}$ via Thom isomorphism. Hence $\operatorname{sign}(D E)=\operatorname{sign}\left(B_{c}\right)$ which finishes the proof of the second part of (4.2).

Now, we assume that $B$ admits a spin structure and we want to calculate $s(P, \phi, g)$. This is more difficult since there is no obvious spin manifold $W$ bounding $P$ with the spin structure $\phi$. Our proof is more indirect and consists of a number of steps. The strategy is to show that it is possible to reduce the calculation for a general $(B, E)$ to a special case where we $d o$ have an explicit spin zero bordism $W$. We will concentrate on the case $k>1$ and discuss the easier case $k=1$ at the end of this section.

Suppose that $W$ is some spin zero bordism for $P$. For the calculation of $s(P, \phi, g)$ via (4.1) it is useful to compare the topological correction term $t(W)$ to $t(D E)$, which we computed above. So we glue $D E$ and $-W$ along their common boundary $P$ to get a closed manifold $D E \cup_{P}-W$ ( $-W$ stands for the manifold $W$ with its orientation reversed). Note that this manifold is oriented, but not spin. Using the fact that $c$ behaves additively when glueing manifolds along their boundaries and that by the signature theorem $t(N)=-\hat{A}(N)$ for a closed manifold $N$ we get

$$
\begin{aligned}
s(P, g) & =\operatorname{ind} D^{+}\left(W, g_{W}\right)+t(W) \\
& =\operatorname{ind} D^{+}\left(W, g_{W}\right)-t\left(D E \cup_{P}-W\right)+t(D E) \\
& =\operatorname{ind} D^{+}\left(W, g_{W}\right)+\hat{A}\left(D E \cup_{P}-W\right)+t(D E) .
\end{aligned}
$$

We observe that ind $D^{+}\left(W, g_{W}\right)$ and $\hat{A}\left(D E \cup_{P}-W\right)$ are well defined without the assumption that the rational Pontrjagin classes of $M$ vanish. Also, the sum ind $D^{+}\left(W, g_{W}\right)+\hat{A}\left(D E \cup_{P}-W\right)$ is independent of the choice of $W$. For those manifolds $M$ with vanishing rational Pontrjagin classes this follows from (4.5), since $s(P, g)$ is independent of $W$. For a general $P$ suppose that $W$, $W^{\prime}$ are two spin manifolds bounding $M$. Then the following equation shows the independence of $W$.

$$
\begin{aligned}
& \text { ind } D^{+}\left(W, g_{W}\right)-\text { ind } D^{+}\left(W^{\prime}, g_{W^{\prime}}\right)=\operatorname{ind} D^{+}\left(W \cup_{P}-W^{\prime}, g_{W} \cup g_{W^{\prime}}\right) \\
& =\hat{A}\left(W \cup_{P}-W^{\prime}\right)=-\hat{A}\left(D E \cup_{P}-W\right)+\hat{A}\left(D E \cup_{P}-W^{\prime}\right) .
\end{aligned}
$$


Here the first equality follows from Remark 2.4. The third equality holds since $W \cup_{P}-W^{\prime}$ is bordant to the disjoint union of $-\left(D E \cup_{P}-W\right)$ and $D E \cup_{P}-W^{\prime}$. Below we show that ind $D^{+}\left(W, g_{W}\right)+\hat{A}\left(D E \cup_{P}-W\right)$ depends only on the class of $(B, E)$ in a suitable bordism group. For a space $X$ let $\Omega_{n}^{\text {spin }}(X)$ be the bordism group of pairs $(M, f)$ consisting of an $n$-dimensional closed spin manifold $M$ and a map $f: M \rightarrow X$. Identifying the complex line bundle $E$ with its classifying map $B \rightarrow B S^{1}$ the pair $(B, E)$ represents an element of $\Omega_{4 k-2}^{\text {spin }}\left(B S^{1}\right)$.

Lemma 4.6. If $k>1$, ind $D^{+}\left(W, g_{W}\right)+\hat{A}\left(D E \cup_{P}-W\right)$ depends only on the bordism class of $(B, E)$ in $\Omega_{4 k-2}^{\mathrm{spin}}\left(B S^{1}\right)$ and hence it defines a homomorphism from the bordism group $\Omega_{4 k-2}^{\text {spin }}\left(B S^{1}\right)$ to $\mathbb{Q}$.

We observe that this result implies that ind $D^{+}\left(W, g_{W}\right)$ is in fact independent of $g=\left(g_{W}\right)_{\mid \partial W}$ (which is assumed to be $S^{1}$-equivariant and to have totally geodesic fibres). We also note that the formula also holds for $k=1$ but we only have a more complicated and indirect proof for this fact which we do not use in this paper.

Proof. We note that ind $D^{+}\left(W, g_{W}\right)$ and $\hat{A}\left(D E \cup_{P}-W\right)$ behave additively under the disjoint union of manifolds. Hence we can always replace $(B, E)$ by a disjoint union of copies of $(B, E)$ and it suffices to show that ind $D^{+}\left(W, g_{W}\right)+$ $\hat{A}\left(D E \cup_{P}-W\right)$ vanishes if $(B, E)$ is zero bordant. So assume that there is a spin manifold $N$ with boundary $B$ such that $E$ extends to a complex line bundle $E_{N}$ over $N$. We choose $W$ to be the sphere bundle $S\left(E_{N}\right)$. Then $\hat{A}\left(D E \cup_{P}-W\right)=0$ since $D E \cup_{P}-W$ is the boundary of the disk bundle $D\left(E_{N}\right)$. Moreover, if we can extend $g_{B}$ to a positive scalar curvature metric $g_{N}$ on $N$ (product metric near the boundary) then the metric $g_{W}$ on $W=S\left(E_{N}\right)$ determined according to (4.3) by $g_{N}$ and a connection on $E_{N}$ has positive scalar curvature by (4.4), provided that the fibres are small enough, and hence ind $D^{+}\left(W, g_{W}\right)$ vanishes.

We note that $s(P, g)$ vanishes if $P$ is the trivial bundle $P=B \times S^{1}$ and $g$ is a product metric, since changing the orientation of $P$ changes the sign of $s(P, g)$ (follows from Definition 2.12) and complex conjugation on $S^{1}$ gives an orientation reversing isometry of $P$. This involution extends to $D E$ and hence also $t(D E)$ is zero. Thus it suffices to construct a positive scalar curvature metric $g_{N}$ on a bordism $N$ between $(B, E)$ and $(B, \mathbb{C})$, where $\mathbb{C}$ stands for the trivial complex line bundle.

Let $N^{\prime}$ be the product of $B$ and the unit interval $I$ and let $g_{N^{\prime}}$ be the product metric on $N^{\prime}$. Then $N^{\prime}$ is a spin bordism between $B$ and itself (of course there is no complex line bundle over $N^{\prime}$ restricting to $E$ on one boundary component and the trivial bundle on the other unless $E$ is trivial). Our goal is to use the positive scalar curvature metric $g_{N^{\prime}}$ to produce a positive scalar curvature metric $g_{N}$ on $N$. We note that the group $\Omega_{4 k-1}^{\text {spin }}$ consists entirely of 2-torsion [ABP]. This implies that (possibly after replacing $(B, E)$ 
by two disjoint copies) $N$ and $N^{\prime}$ are spin bordant relative boundary, i.e. there is a spin manifold $V$ with boundary $N \cup N^{\prime}$ (glued along their common boundary). This implies that $N$ can be obtained from $N^{\prime}$ by surgeries in the interior and hence by the surgery results of [GL1], resp. [SY], the manifold $N$ admits a positive scalar curvature metric $g_{N}$, provided that the surgeries needed are all of codimension $\geq 3$.

We observe that a surgery of codimension $i$ correponds to an $(n-i)$-handle of the pair $\left(V, N^{\prime}\right)$ with $n=\operatorname{dim} V$. There is a handle decomposition of $\left(V, N^{\prime}\right)$ without $(n-i)$-handles for $i \leq 2$, provided that $H_{n-i}\left(V, N^{\prime} ; \mathbb{Z}\right) \cong$ $H^{i}(V, N ; \mathbb{Z})$ vanishes for $i \leq 2$ [Mi]. This is the case if the inclusion $N \rightarrow V$ is a 2-equivalence; i.e. it induces an isomorphism on homotopy groups $\pi_{0}, \pi_{1}$ and a surjection on $\pi_{2}$. We claim that we can always modify $\left(N, E_{N}\right)$ and $V$ such that the inclusion is a 2-equivalence. First we do 0 - and 1-surgeries on $N$ in order to make the map $N \rightarrow B S^{1}$ classifying $E_{N}$ a 2-equivalence. Then we make $V$ 2-connected by $i$-surgeries, $i \leq 2$ (here it is important that $V$ is spin since otherwise there might be a class in $\pi_{2}(V)$ which we cannot kill by surgery since it is represented by an embedded 2-sphere with nontrivial normal bundle). After these modifications the inclusion $N \rightarrow V$ is clearly a 2-equivalence and this finishes the proof of Lemma 4.6.

Our next goal is to show that a multiple of each class in $\Omega_{4 k-2}^{\text {spin }}\left(B S^{1}\right)$ can be represented by total spaces of fibre bundles with fibre $\mathbb{C P}^{1}$. More precisely, let

$$
\mathbb{C P}^{1}=S U(2) / S^{1} \rightarrow B S^{1} \stackrel{\pi}{\longrightarrow} B S U(2)
$$

be the fibre bundle with fibre $\mathbb{C P}^{1}$ induced by the standard embedding of $S^{1}$ as diagonal matrices in $S U(2)$. Let $\tau$ be the tangent bundle along the fibres. We note that $\tau$ is spin since its restriction to a fibre is spin. Hence there is a corresponding transfer homomorphism of spin bordism groups [Bo]

$$
\pi^{!}: \Omega_{n}^{\mathrm{spin}}(B S U(2)) \rightarrow \Omega_{n+2}^{\mathrm{spin}}\left(B S^{1}\right),
$$

defined geometrically as follows. Given a closed spin manifold $X$ and a map $h: X \rightarrow B S U(2)$ let $\hat{X} \rightarrow X$ be the pull back of the bundle (4.7) via $h$ and let $\hat{h}: \hat{X} \rightarrow B S^{1}$ be the map covering $h$. The transfer map $\pi^{!}$maps the bordism class of $(X, h)$ to the bordism class of $(\hat{X}, \hat{h})$ (note that a spin structure on $X$ induces a spin structure on $\hat{X}$ since the tangent bundle along the fibres is spin).

Lemma 4.9. In degrees $n \equiv 0 \bmod 4$ the transfer $\pi^{!}$is a rational isomorphism. Proof. In homotopy theoretic terms the transfer map $\pi^{!}$can be described as follows [Bo]. Let $M(-\tau)$ be the Thom spectrum of the inverse of $\tau$. Associated to the fibre bundle (4.7) there is a "Thom" map $T(\pi)$ from the suspension spectrum of $B S U(2)$ to the desuspension $\Sigma^{-2} M(-\tau)$. Then $\pi^{!}$is the composition

$$
\Omega_{n}^{\mathrm{spin}}(B S U(2)) \stackrel{T(\pi)_{*}}{\longrightarrow} \Omega_{n}^{\mathrm{spin}}\left(\Sigma^{-2} M(-\tau)\right) \stackrel{\Phi}{\cong} \Omega_{n+2}^{\mathrm{spin}}\left(B S^{1}\right)
$$


where $\Phi$ is the Thom isomorphism in spin bordism. Hence it suffices to show that $T(\pi)_{*}$ is a rational isomorphism for $n \equiv 0 \bmod 4$. We note that there is a natural isomorphism

$$
\Omega_{*}^{\text {spin }}(X) \otimes \mathbb{Q} \cong H_{*}(X ; \mathbb{Q}) \otimes \Omega_{*}^{\text {spin }} .
$$

Moreover, $\Omega_{*}^{\text {spin }} \otimes \mathbb{Q}$ is a polynomial ring with generators of degree $0 \bmod 4$. This implies that it suffices to show that the map induced by $T(\pi)$ in rational homology is an isomorphism in degrees $n \equiv 0 \bmod 4$. Dually, it suffices to show that the cohomology transfer

$$
\pi_{!}: H^{n+2}\left(B S^{1} ; \mathbb{Z}\right) \stackrel{\Phi}{\cong} H^{n+2}\left(\Sigma^{-2} M(-\tau) ; \mathbb{Z}\right) \stackrel{T(\pi)^{*}}{\longrightarrow} H^{n}(B S U(2) ; \mathbb{Z})
$$

is a rational isomorphism for $n \equiv 0 \bmod 4$ ( $\Phi$ is here the Thom isomorphism in cohomology). This transfer can be described explicitly as follows. Recall that $H^{*}\left(B S^{1} ; \mathbb{Z}\right) \cong \mathbb{Z}[x]$, where $x$ is an element of degree 2 which restricts to a generator of the second cohomology of the fibre of (4.7). Hence the LerayHirsch theorem implies that each element $z$ in $H^{*}\left(B S^{1} ; \mathbb{Z}\right)$ can be written uniquely in the form $z=\pi^{*}(a)+\pi^{*}(b) \cup x$ with $a, b \in H^{*}(B S U(2) ; \mathbb{Z}) \cong \mathbb{Z}[y]$ ( $y$ has degree 4 ).

It follows from the Serre spectral sequence description of $\pi_{1}$ [Bo, Chapter $\mathrm{V}, 6.14]$ that $\pi_{!}(z)=b$. Hence $\pi_{!}\left(x^{2 k+1}\right)=y^{k}$ which proves Lemma 4.9.

Lemma 4.9 shows it suffices to determine ind $D^{+}\left(W, g_{W}\right)+\hat{A}\left(D E \cup_{P}-W\right)$ for pairs $(B, E)$ in the image of $\pi$ !. So we can assume that $B$ is the total space of a $\mathbb{C P}^{1}$-bundle $\hat{X} \rightarrow X$ which is the pull back of (4.7) via a map $h: X \rightarrow B S U(2)$ and that $E$ is classified by $\hat{h}: \hat{X} \rightarrow B S^{1}$. In more concrete terms: Let $V$ be the quaternionic line bundle classified by $h$. Then

(1) $B$ is the complex projective bundle $\mathbb{C P}(V)$.

(2) $E$ is the tautological complex line bundle over $\mathbb{C P}(V)$ (whose fibre over a point in $\mathbb{C P}(V)$, i.e. a complex line in $V$, consists of all vectors in that line).

(3) $P$ is the sphere bundle $S E$ which can be identified with the sphere bundle $S V$.

(4) $W$ can be chosen to be the disk bundle $D V$ (this is spin!).

(5) $D E \cup_{P}-W$ can be identified with the complex projective bundle $\mathbb{C P}(V \oplus \mathbb{C})$, where $\mathbb{C}$ is the trivial complex line bundle.

(6) The Riemannian metric $g$ on $P=S V$ can be chosen to be the $S^{3}$ equivariant metric with totally geodesic fibres associated by (4.3) to a metric $g_{X}$ on $X$, a connection $\theta$ on $V$, and the standard metric (suitably scaled) on the fibre $S^{3}$.

Lemma 4.10. (1) ind $D^{+}\left(D V, g_{D V}\right)=0$ for any metric $g_{D V}$ on $D V$ which restricts to $g$ on the boundary and is a product metric in a collar neighbourhood of the boundary.

(2) $\hat{A}(\mathbb{C P}(V \oplus \mathbb{C}))=\left\langle\hat{A}(T \mathbb{C P}(V))\left(\frac{1}{2 \sinh (c / 2)}-\frac{1}{2 \tanh (c / 2)}\right),[\mathbb{C P}(V)]\right\rangle$.

The proof of the first part is completely analogous to the proof of the first part of (4.2). We defer the characteristic class calculation necessary for the proof of the second part to the next section. 
Formula (4.5) shows that combining this lemma with the calculation of $t(D E)$ in (4.2) gives the desired formula for $s(P, \phi, g)$. This finishes the proof of Theorem 3.11 for $k>1$.

The case $k=1$ is so far easier as the only oriented 2-manifold with positive scalar curvature metric is $S^{2}$. Since the space of positive scalar curvature metrics on $S^{2}$ is connected (this follows from uniformization theory), we can assume that the metric is the standard round metric on $S^{2}$. Then, if the $S^{1}$ bundle is the Hopf bundle, Theorem 3.11 follows from Lemma 4.11. The case of arbitrary bundles over $S^{2}$ follows from this case by considering $N=D^{3}$ with two holes. By [GL] there is a metric with positive scalar curvature on $N$, which near the three boundary $S^{2}$ 's is a product metric of the standard round metric. Now, consider the $S^{1}$-bundle over $N$ which on the boundary of the holes has first Chern class $k$ and $l$. If we shrink the metric on the total space $W$ enough, $W$ admits a metric of positive scalar curvature extending the metrics of our type on the boundary components. Thus the index of the Dirac operator on $W$ vanishes and we can reduce Theorem 3.11 by (4.5) to the case of the Hopf bundle.

\section{MULTIPLICATIVE GENERA AND FIBRE BUNDLES}

The goal of this section is to prove the second part of (4.10), i.e. to calculate the $\hat{A}$-genus of the complex projective bundle $\mathbb{C P}(V \oplus \mathbb{C})$. Here $V \oplus \mathbb{C}$ is a 3-dimensional complex vector bundle over a spin manifold $X$, namely the sum of a quaternionic line bundle $V$ and the trivial complex line bundle $\mathbb{C}$. We remark that $\mathbb{C P}(V \oplus \mathbb{C})$ has a positive scalar curvature metric, but this does not imply the vanishing of the $\hat{A}$-genus, since it is not a spin manifold. Likewise, the multiplicativity of the $\hat{A}$-genus for fibre bundles [AH] does not apply in our situation since the fibre $\mathbb{C P}^{2}$ is not spin. In the following calculation of $\hat{A}(\mathbb{C P}(V \oplus \mathbb{C}))$ we do not use special properties of the $\hat{A}$-genus and so we consider a general multiplicative genus $K$ [MS, $\S 19]$. Also, we first discuss the $K$-genus of a general bundle with fibre a homogeneous space $G / H$ and structure group $G$ and then specialize to $\mathbb{C P}^{2}$-bundles.

Let $G$ be a compact connected Lie group and let $i: H \rightarrow G$ be the inclusion of a closed connected subgroup. This inclusion induces a projection map of classifying spaces and we denote by $\tau$ the tangent bundle along the fibres of the fibre bundle

$$
G / H \rightarrow B H \stackrel{B i}{\longrightarrow} B G
$$

Lemma 5.2. Let $K$ be a multiplicative genus, let $X$ be an oriented manifold and let $\pi: \hat{X} \rightarrow X$ be the pull back of (5.1) via a map $g: X \rightarrow B G$. Then

$$
K(\hat{X})=\left\langle K(T X) g^{*}(B i), K(\tau),[X]\right\rangle .
$$

Proof. The tangent bundle $T \hat{X}$ is isomorphic to $\pi^{*} T X \oplus \hat{g}^{*}(\tau)$, where $\hat{g}: \hat{X} \rightarrow$ $B H$ is the map covering $g$. The multiplicativity of $K$ then implies $K(T \hat{X})=$ $\pi^{*} K(T X) \hat{g}^{*} K(\tau)$. Hence

$$
K(\hat{X})=\left\langle\pi_{!} K(T \hat{X}),[X]\right\rangle=\left\langle K(T X) \pi_{!}\left(\hat{g}^{*} K(\tau)\right),[X]\right\rangle,
$$


where the second equality follows from the fact that $\pi_{!}\left(\pi^{*} x \hat{x}\right)=x \pi_{!}(\hat{x})$ for elements $x, \hat{x}$ in the cohomology of $X$, respectively $\hat{X}$. This implies the lemma, since $\pi_{!}\left(\hat{g}^{*} K(\tau)\right)=g^{*}(B i)_{!} K(\tau)$ by the naturality of the transfer homomorphism.

To use Lemma 5.2 we need to determine $(B i), K(\tau)$ which is the "equivariant $K$-genus" of $G / H$. Recall that the inclusion of a maximal torus $j: T \rightarrow G$ induces an injective homomorphism (cf. $[\mathrm{BH}])$

$$
B j^{*}: H^{*}(B G ; \mathbb{Q}) \rightarrow H^{*}(B T ; \mathbb{Q}) \text {. }
$$

Moreover, the image is the fixed point set $H^{*}(B T ; \mathbb{Q})^{W(G)}$ of the action of the Weyl group $W(G)$. We will use $B j^{*}$ to identify elements of $H^{*}(B G ; \mathbb{Q})$ with their image in $H^{*}(B T ; \mathbb{Q})$. Consequently, the map $B j^{*}$ (and its analogue for a maximal rank subgroup $H$ ) will be suppressed in the formulas below. Recall that the cohomology of $B T$ is a polynomial ring. It is convenient to construct explicit generators in the following way. Let $\alpha$ be a homomorphism from the torus to $S^{1}$. Then $\alpha$ determines a complex line bundle over $B T$ (the vector bundle associated to $\alpha$ regarded as 1 -dimensional complex representation of $T$ ) and a cohomology class in $H^{2}(B T ; \mathbb{Z})$ (the first Chern class of that line bundle). Abusing notation we will again write $\alpha$ for this cohomology class. If $x_{1}, \ldots, x_{l}$ is a basis of $\operatorname{Hom}\left(T, S^{1}\right)$ then $H^{*}(B T ; \mathbb{Q})$ is the polynomial ring generated by $x_{1}, \ldots, x_{l}$. [BH].

The proof of the next proposition is based on results of Borel and Hirzebruch

Proposition 5.3. Let $K$ be a multiplicative genus with characteristic power series $Q(x)=x / f(x)$, where $f(x)=x+\cdots$ is an odd power series. Let $G$ be a compact connected Lie group and let $i: H \rightarrow G$ be the inclusion of a closed connected subgroup of maximal rank. Let $\alpha_{1}, \ldots, \alpha_{m+n}$ be a set of roots of $G$ which contains for each root $\alpha$ exactly one of the roots $\alpha,-\alpha$. Assume that $\alpha_{1}, \ldots, \alpha_{m}$ are roots of $H$ and $\alpha_{m+1}, \ldots, \alpha_{m+n}$ are complementary roots, i.e. roots of $G$ which are not roots of $H$. Then

$$
(B i)_{!}(y)=\sum_{w \in W(G) / W(H)} w\left(\frac{y}{\alpha_{m+1} \cdots \alpha_{m+n}}\right) \quad \text { for } y \in H^{*}(B H ; \mathbb{Q}),
$$

$$
(B i)_{!}(K(\tau))=\frac{1}{f\left(\alpha_{1}\right) \cdots f\left(\alpha_{m+n}\right)} \sum_{w \in W(G) / W(H)} \operatorname{sgn}(w) w\left(f\left(\alpha_{1}\right) \cdots f\left(\alpha_{m}\right)\right),
$$

where $\operatorname{sgn}(w) \in\{ \pm 1\}$ is defined by $w\left(\alpha_{1} \cdots \alpha_{m+n}\right)=\operatorname{sgn}(w) \alpha_{1} \cdots \alpha_{m+n}$.

Here the fractional expressions are to be considered as elements in the ring obtained from the polynomial ring $H^{*}(B T ; \mathbb{Q})$ by adjoining the inverses of the roots of $G$. We note that $(B i)$, ("integration over the fibre") on the left-hand side of the above equations depends on the choice of an orientation for $\tau$, while the right-hand side depends on the choice of signs for $\alpha_{m+1}, \ldots, \alpha_{m+n}$. The orientation convention making the above formulas correct is the same as 
in [BH], namely we have to pick the orientation of $\tau$ such that its Euler class is $\alpha_{m+1} \cdots \alpha_{m+n}$ (it is not hard to see that the Euler class of $\tau$ is $\pm \alpha_{m+1} \cdots \alpha_{m+n}$; cf. proof of part (2) of the proposition). We observe that the Euler class of $\tau$ is an element of $H^{*}(B H)$ and hence invariant under $W(H)$ which shows that the right-hand sides are well defined, i.e. independent of the choice of coset representatives for $W(G) / W(H)$.

Proof of Proposition 5.3. Let $B j: B T \rightarrow B G$ be the projection map induced by the inclusion of a maximal torus of $G$. Then by [BH, Theorem 20.3]

$$
\sum_{w \in W(G)} \operatorname{sgn}(w) w(x)=(B j)_{!}(x) \alpha_{1} \cdots \alpha_{m+n} \quad \text { for } \quad x \in H^{*}(B T) .
$$

Dividing by $\alpha_{1} \cdots \alpha_{m+n}$ and using the definition of $\operatorname{sgn}(w)$ we get

$$
(B j)_{!}(x)=\sum_{w \in W(G)} w\left(\frac{x}{\alpha_{1} \cdots \alpha_{m+n}}\right) \quad \text { for } \quad x \in H^{*}(B T) .
$$

In particular,

$$
(B j)_{!}\left(z \alpha_{1} \cdots \alpha_{m+n}\right)=|W(G)| z \quad \text { for } \quad z \in H^{*}(B G),
$$

where $|W(G)|$ is the order of $W(G)$. Our assumption that $G$ and $H$ have the same rank implies that the inclusion $j: T \rightarrow G$ factors in the form $T \stackrel{k}{\longrightarrow} H \stackrel{i}{\longrightarrow}$ $G$ and hence $(B j)_{!}=(B i)_{!}(B k)_{!}$. Applying (5.4) to the element $x=y \alpha_{1} \cdots \alpha_{m}$ with $y \in H^{*}(B H)$ we get

$$
\begin{aligned}
(B j)_{!}\left(y \alpha_{1} \cdots \alpha_{m}\right) & =\sum_{w \in W(G)} w\left(\frac{y}{\alpha_{m+1} \cdots \alpha_{m+n}}\right) \\
& =|W(H)| \sum_{w \in W(G) / W(H)} w\left(\frac{y}{\alpha_{m+1} \cdots \alpha_{m+n}}\right) .
\end{aligned}
$$

The second equality follows from the fact that elements of $W(H)$ act trivially on $y$ and $\alpha_{m+1} \cdots \alpha_{m+n}$. On the other hand,

$$
(B i)_{!}(B k)_{!}\left(y \alpha_{1} \cdots \alpha_{m}\right)=|W(H)|(B i)_{!}(y)
$$

by applying (5.5) to the subgroup $H$, which proves part (1).

To prove part (2) we decompose the Lie algebra of $G$ in the form $\mathfrak{g}=$ $\mathfrak{h} \oplus \mathfrak{h}^{\perp}$, where $\mathfrak{h}$ is the Lie algebra of $H$ and $\mathfrak{h}^{\perp}$ is an $H$-invariant complement. We note that $\tau$ is the vector bundle associated to $\mathfrak{h}^{\perp}$. In particular, $(B j)^{*}(\tau)$ is isomorphic to the sum of the complex line bundles over $B T$ associated to $\alpha_{m+1}, \ldots, \alpha_{m+n}$. It follows that the Euler class of $(B j)^{*}(\tau)$ is $\pm \alpha_{m+1} \cdots \alpha_{m+n} \in H^{*}(B T)$. Moreover,

$$
K(\tau)=\frac{\alpha_{m+1} \cdots \alpha_{m+n}}{f\left(\alpha_{m+1}\right) \cdots f\left(\alpha_{m+n}\right)}
$$


and hence

$$
\begin{aligned}
(B i)_{!}(K(\tau)) & =\sum_{w \in W(G) / W(H)} w\left(\frac{1}{f\left(\alpha_{m}\right) \cdots f\left(\alpha_{m+n}\right)}\right) \\
& =\frac{1}{f\left(\alpha_{1}\right) \cdots f\left(\alpha_{m+n}\right)} \sum_{w \in W(G) / W(H)} \operatorname{sgn}(w) w\left(f\left(\alpha_{1}\right) \cdots f\left(\alpha_{m}\right)\right),
\end{aligned}
$$

which proves the second part of Proposition 5.3.

Now we specialize to the case where $G / H$ is $\mathbb{C P}^{2}$, i.e. we consider the fibre bundle

$$
\mathbb{C P}^{2}=U(3) /(U(2) \times U(1)) \rightarrow B(U(2) \times U(1)) \stackrel{B i}{\longrightarrow} B U(3),
$$

where the inclusion $i: H \rightarrow G$ maps $(A, B) \in U(2) \times U(1)$ to the three-bythree block matrix with $A$ in the upper left, $B$ in the lower right corner, and zeros everywhere else.

The maximal torus of $U(3)$ is 3-dimensional. The inclusion $j: T \rightarrow U(3)$ is given by mapping $\left(z_{1}, z_{2}, z_{3}\right) \in T$ to the diagonal matrix with entries $\left(z_{1}, z_{2}, z_{3}\right)$. Let $x_{i}: T \rightarrow S^{1}$ be the projection on the $i$ th factor, $i=1,2,3$. The Weyl group of $U(3)$ is the symmetric group $\Sigma_{3}$ which acts on the $x_{i}$ 's by permuting them. The Weyl group of $U(2) \times U(1)$ is the subgroup $\Sigma_{2}$ fixing $x_{3}$. The roots of $U(2) \times U(1)$ are $\pm\left(x_{1}-x_{2}\right)$ and the roots of $U(3)$ are $\pm\left(x_{i}-x_{j}\right)$ for $1 \leq i<j \leq 3$. So we can choose the $\alpha$ 's to be of the form $x_{i}-x_{j}, 1 \leq i<j \leq 3$. Moreover we can choose the coset representatives $w$ of the quotient of the Weyl groups to be the cyclic permutations of $1,2,3$. Then $\operatorname{sgn}(w)=1$ and using part (2) of Proposition 5.3 we get

$$
(B i)_{!}(K(\tau))=\frac{f\left(x_{1}-x_{2}\right)+f\left(x_{2}-x_{3}\right)+f\left(x_{3}-x_{1}\right)}{f\left(x_{1}-x_{2}\right) f\left(x_{2}-x_{3}\right) f\left(x_{1}-x_{3}\right)} \text {. }
$$

Now we want to apply this to the complex projective bundle $\mathbb{C P}(V \oplus \mathbb{C}) \rightarrow X$, where $V$ is a quaternionic line bundle classified by $h: X \rightarrow B S U(2)$. Combining Lemma 5.2 and (5.7) we get a calculation of the $K$-genus of $\mathbb{C P}(V \oplus \mathbb{C})$ in terms of characteristic numbers of $X$. For the proof of the second part of Lemma 4.10, however, we need a calculation in terms of characteristic numbers of $\mathbb{C P}(V)$. The answer is the following.

Lemma 5.8. Let $K$ be a multiplicative genus with characteristic power series $x / f(x)$. Then

$$
K(\mathbb{C P}(V \oplus \mathbb{C}))=\left\langle K(T \mathbb{C P}(V))\left(\frac{1}{f(c)}-\frac{f(2 c)}{2 f(c)^{2}}\right),[\mathbb{C P}(V)]\right\rangle,
$$

where $c$ is the first Chern class of the canonical complex line bundle over $\mathbb{C P}(V)$.

Specializing $K$ to the $\hat{A}$-genus for which $f(x)=2 \sinh (x / 2)$ we get part (2) of Lemma 4.10.

Proof of Lemma 5.8. We note that the $\mathbb{C P}^{2}$-bundle $\mathbb{C P}(V \oplus \mathbb{C}) \rightarrow X$ is the pull back of the bundle (5.6) via the composition of the map $h: X \rightarrow B S U(2)$ 
classifying $V$ and $B k: B S U(2) \rightarrow B U(3)$, where $k$ maps $A \in S U(2)$ to the block matrix with $A$ in the upper left corner, 1 in the lower right corner, and zeros everywhere else.

Applying Lemma 5.2 we get

$$
\begin{aligned}
K(\mathbb{C P}(V \oplus \mathbb{C})) & =\left\langle K(T X) h^{*}(B k)^{*}(B i)_{!} K(\tau),[X]\right\rangle \\
& =\left\langle q^{*}\left(K(T X) h^{*}(B k)^{*}(B i)_{!} K(\tau)\right) c,[\mathbb{C P}(V)]\right\rangle,
\end{aligned}
$$

where $q$ is the projection map $q: \mathbb{C P}(V) \rightarrow X$ and $c$ is the first Chern class of the tautological complex line bundle over $\mathbb{C P}(V)$. The second equation follows from the fact that $q_{!}(c)=1$ and hence $q_{!}\left(q^{*} x c\right)=x$ for all elements $x$ in the cohomology of $X$.

We note that the $\mathbb{C P}^{1}$-bundle $q: \rightarrow X$ is the pull back via $h: X \rightarrow B S U(2)$ of the bundle

$$
\mathbb{C P}^{1}=S U(2) / S^{1} \rightarrow B S^{1} \stackrel{B i^{\prime}}{\longrightarrow} B S U(2),
$$

where $i^{\prime}$ maps $z \in S^{1}$ to the diagonal matrix in $S U(2)$ with entries $z, z^{-1}$. Hence the tangent bundle $T \mathbb{C P}(V)$ is isomorphic to $q^{*} T X \oplus \hat{h}^{*}\left(\tau^{\prime}\right)$, where $\tau^{\prime}$ is the tangent bundle along the fibres of $(5.10)$ and $\hat{h}: \mathbb{C P}(V) \rightarrow B S^{1}$ is the map covering $h$. It follows that $K(T \mathbb{C P}(V))=q^{*} K(T X) \hat{h}^{*} K\left(\tau^{\prime}\right)$ and hence

$$
\begin{aligned}
& q^{*}\left(K(T X) h^{*}(B k)^{*}(B i), K(\tau)\right) \\
& \quad=K(T \mathbb{C P}(V)) \hat{h}^{*}\left(K\left(\tau^{\prime}\right)^{-1}\left(B i^{\prime}\right)^{*}(B k)^{*}(B i)_{!} K(\tau)\right) .
\end{aligned}
$$

If $x$ is the generator of $H^{*}\left(B S^{1} ; \mathbb{Z}\right)$ the homomorphism $\left(B i^{\prime}\right)^{*}(B k)^{*}$ maps $x_{1}$ to $x, x_{2}$ to $-x$, and $x_{3}$ to zero. Using (5.7) and the fact that $f$ is an odd power series we obtain

$$
\left(B i^{\prime}\right)^{*}(B k)^{*}(B i)_{!} K(\tau)=\frac{f(2 x)-2 f(x)}{-f(2 x) f(x)^{2}} .
$$

The tangent bundle along the fibres of $(5.10)$ is a complex line bundle with first Chern class $2 x$ and hence $K\left(\tau^{\prime}\right)=\frac{2 x}{f(2 x)}$. It follows that

$$
K\left(\tau^{\prime}\right)^{-1}\left(B i^{\prime}\right)^{*}(B k)^{*}(B i), K(\tau)=\frac{1}{x f(x)}-\frac{f(2 x)}{2 x f(x)^{2}} .
$$

Putting this together with (5.9) and (5.11) and noting that $\hat{h}^{*}$ maps $x$ to $c$ finishes the proof of Lemma 5.8 .

\section{ACKNOWLEDGMENTS}

We would like to thank Rainer Jung for numerous useful discussions.

\section{REFERENCES}

[ABP] D.W. Anderson, E. H. Brown, Jr., and F. P. Peterson, The structure of the spin cobordism ring, Ann. of Math. (2) 86 (1967), 271-298.

[AH] M.F. Atiyah and F. Hirzebruch, Spin manifolds and group actions, Essays in Topology and Related Topics, Proceedings, Springer, 1970, pp. 18-28.

[ÄPS1] M. F. Atiyah, Y. Patodi and I. Singer, Spectral asymmetry and Riemannian geometry. I, Math. Proc. Cambridge Philos. Soc. 77 (1975), 43-69. 
[APS2] _ Spectral asymmetry and Riemannian geometry. II, Math. Proc. Cambridge Philos. Soc. 78 (1975), 405-432.

[AW] S. Aloff and N. Wallach, An infinite family of distinct 7-manifolds admitting positively curved Riemannian structures, Bull. Amer. Math. Soc. 81 (1975), 93-97.

[Be] A. L. Besse, Einstein manifolds, Spinger-Verlag, Berlin and New York, 1986.

[Bo] J. M. Boardman, Stable homotopy theory, mimeographed notes, Warwick (1966).

[BH] A. Borel and F. Hirzebruch, Characteristic classes and homogeneous spaces, Amer. J. Math. 80 (1958), 458-538;II, Amer. J. Math. 81 (1959), 315-382.

[Ca] R. Carr, Constructions of manifolds of positive scalar curvaature, Trans. Amer. Math. Soc. 307 (1988), 63-74.

[CS] S. Cappell and J. Shaneson, The topology of linear representations of groups and subgroups, Amer. J. Math 104 (1982), 773-778.

[Ga] P. Gajer, Riemannian metrics of positive scalar curvature on compact manifolds with boundary, Ann. Global Anal. Geom. 5 (1987), 179-191.

[Gr] M. Gromov, Curvature, diameter and Betti numbers, Comment. Math. Helv. 56 (1981), 179-195.

[GL1] M. Gromov and H. B. Lawson, Jr., The classification of simply connected manifolds of positive scalar curvature, Ann. of Math. (2) 111 (1980), 423-434.

[GL2] , Positive scalar curvature and the Dirac operator on complete Riemannian manifolds, Publ. Math. Inst. Hautes Études Sci. 58 (1983), 83-196.

[Ha1] R. Hamilton, Three-manifolds with positive Ricci cirvature, J. Differential Geom. 17 (1982), 255-306.

[Ha2] _ Four-manifolds with positive curvature operator, J. Differential Geom. 24 (1986), 153-179.

[Hir] F. Hirzebruch, Neue topologische Methoden in der algebraischen Geometrie, Grundlehren Math. Wiss., Bd. 131, Springer, 1966.

[Hit] N. Hitchin, Harmonic spinors, Adv. in Math. 14 (1974), 1-55.

[Kr] M. Kreck, Bordism of diffeomorphisms and related topics, Lecture Notes in Math., vol. 1069, Springer-Verlag, New York and Berlin, 1984.

[KS1] M. Kreck and S. Stolz, A diffeomorphism classification of 7-dimensional homogeneous Einstein manifolds with $S U(3) \times S U(2) \times U(1)$-symmetry, Ann. of Math. (2) 127 (1988), 373-388.

[KS2] __ Some nondiffeomorphic homeomorphic homogeneous 7-manifolds with positive sectional curvature, J. Differential Geom. 33 (1991), 465-486.

[Li] A. Lichnerowicz, Spineurs harmoniques, C. R. Acad. Sci. Paris Sér. A-B 257 (1963), 7-9.

[Mi] J. W. Milnor, Lectures on the h-cobordism theorem, Princeton Univ. Press, Princeton, NJ, 1965.

[MS] J. W. Milnor and J. D. Stasheff, Characteristic classes, Ann. of Math. Stud., no. 76, Princeton Univ. Press, Princeton, NJ, 1974.

[Oc] S. Ochanine, Signature modulo 16, invariants de Kervaire généralisé et nombres caractéristiques dans la $K$-théorie reélle, Bull. Soc. Math. France 5 (1981).

[SY] R. Schoen and S.-T. Yau, On the structure of manifolds with positive scalar curvature, Manuscripta Math. 28 (1979), 159-183.

[St] S. Stolz, Simply connected manifolds of positive scalar curvature, Ann. of Math. (2) 136 (1992), 511-540.

[WZ] M. Wang and W. Ziller, Einstein metrics with positive scalar curvature, Curvature and Topology of Riemannian Manifolds, Proceedings, Lecture Notes in Math., vol. 1201, SpringerVerlag, New York and Berlin, 1986, pp. 319-336.

[Wi] E. Witten, Search for a realistic Kaluza-Klein theory, Nuclear Phys. B 186 (1981), 412-428. 
Abstract. For a closed manifold $M$ let $\mathfrak{R}_{\text {sec }}^{+}(M)$ (resp. $\mathfrak{R}_{\mathrm{Ric}}^{+}(M)$ ) be the space of Riemannian metrics on $M$ with positive sectional (resp. Ricci) curvature and let $\operatorname{Diff}(M)$ be the diffeomorphism group of $M$, which acts on these spaces. We construct examples of 7-dimensional manifolds for which the moduli space $\mathfrak{R}_{\mathrm{sec}}^{+}(M) / \operatorname{Diff}(M)$ is not connected and others for which $\mathfrak{R}_{\mathrm{Ric}}^{+}(M) / \operatorname{Diff}(M)$ has infinitely many connected components. The examples are obtained by analyzing a family of positive sectional curvature metrics on homogeneous spaces constructed by Aloff and Wallach, on which $S U(3)$ acts transitively, respectively a family of positive Einstein metrics constructed by Wang and Ziller on homogeneous spaces, on which $S U(3) \times S U(2) \times U(1)$ acts transitively.

Max-Planck-Institut für Mathematik, Gottfried-Claren-Strasse 26, 5300 Bonn 3, Germany and Fachbereich Mathematik, Universität Mainz, 6500 Mainz, Germany

Current address: Johannes Gutenberg Universität Mainz, Fachbereich Mathematik, Staudingerweg 9, 6500 Mainz, Germany

E-mail address: kreck@topologie.mathematik.uni-mainz.de

Department of Mathematics, University of Notre Dame, Notre Dame, Indiana 46556 E-mail address: stolz.1@nd.edu 